\title{
Name-passing calculi: from fusions to preorders and types (Appendix)
}

\author{
Daniel Hirschkoff, Jean-Marie Madiot \\ ENS Lyon, U. de Lyon, CNRS, INRIA, UCBL \\ $\{$ daniel.hirschkoff, jeanmarie.madiot $\} @$ ens-lyon.fr
}

\author{
Davide Sangiorgi \\ University of Bologna and INRIA \\ davide.sangiorgi@cs.unibo.it
}

This is the appendix of the paper "Name-passing calculi: from fusions to preorders and types" (D Hirschkoff, JM. Madiot, D. Sangiorgi), to appear in LICS'2013.

\section{APPENDIX}

\section{A. Reduction-closed barbed congruence (Section II)}

Definition 28 (Reduction-closed barbed congruence). Let $\mathcal{L}$ be a process calculus, in which a reduction relation $\longrightarrow_{\mathcal{L}}$ and barb predicates $\downarrow_{a}^{\mathcal{L}}$, for each a in a given set of names, have been defined.

A relation $\mathcal{R}$ on the processes of $\mathcal{L}$ is context-closed if $P \mathcal{R} Q$ implies $C[P] \mathcal{R} C[Q]$, for each context $C$ of $\mathcal{L}$; the relation is barb-preserving if for any name a, $P \downarrow_{a}^{\mathcal{L}}$ implies $Q \downarrow_{a}^{\mathcal{L}}$; it is reduction-closed if whenever $P \longrightarrow_{\mathcal{L}} P^{\prime}$, there is $Q^{\prime}$ s.t. $Q \longrightarrow_{\mathcal{L}} Q^{\prime}$ and $P^{\prime} \mathcal{R} Q^{\prime}$.

Then reduction-closed barbed congruence in $\mathcal{L}$, written $\simeq_{\mathcal{L}}$, is the largest symmetric relation on the processes of $\mathcal{L}$ that is context-closed, reduction-closed, and barb-preserving.

\section{B. Proofs of impossibility results (Section III)}

Statement of Theorem 3: A typed calculus with fusions that is plain and supports narrowing has trivial subtyping.

Proof Sketch: We define the following active context:

$$
E \triangleq(\boldsymbol{\nu} c b)(\bar{u} b|u c| \bar{v} a|v c|[\cdot]) .
$$

Note that in $E$ we only use $b$ as an output object. The intention is that, given some process $P$, and $u, v, c$ some fresh names, $E[P]$ should reduce to $P\{a / b\}$. Indeed, by applying hypothesis (2) twice, we have

$$
\begin{aligned}
E[P] & =(\boldsymbol{\nu} b c)(\bar{u} b|\bar{v} a| u c|v c| P) \\
& \Longrightarrow(\boldsymbol{\nu} b)(\bar{v} a|v b| P\{b / c\}) \\
& =(\boldsymbol{\nu} b)(\bar{v} a|v b| P) \\
& \Longrightarrow P\{a / b\} .
\end{aligned}
$$

Suppose $U \leq T$, we show $\Gamma, a: T \vdash P$ iff $\Gamma, a: U \vdash P$. The implication from left to right is narrowing. To prove the right to left implication, suppose $\Gamma, a: U \vdash P$, and prove $\Gamma, a: T \vdash P$. By injective name substitution we have $\Gamma, b$ : $U \vdash P\{b / a\}$ for some fresh $b$.
In the typing environment $\Gamma, b: U, u: \sharp T, v: \sharp T, c: T, a: T$ the process $\bar{u} b$ is well-typed thanks to narrowing and weakening, hence so is $(\bar{u} b|u c| \bar{v} a|v c| P\{b / a\})$. By the restriction rule we get $\Gamma, a: T, u: \sharp T, v: \sharp T \vdash E[P\{b / a\}]$, the latter reducing to $P\{b / a\}\{a / b\}$ by (4). Since $b$ has been taken fresh, $P\{b / a\}\{a / b\}=P$. Hence, by Subject Reduction, $\Gamma, a: T, u: \sharp T, v: \sharp T \vdash P$. We finally deduce $\Gamma, a: T \vdash P$ by Strengthening.

Statement of Theorem 4: Suppose a typed calculus with fusions is plain and there is at least one prefix $\alpha$ with object $b$, different from the subject, and there are two types $S$ and $T$ such that $S \leq T$ and one of the following forms of narrowing holds for all $\Gamma$ :

1) whenever $\Gamma, b: T \vdash \alpha$. 0 , we also have $\Gamma, b: S \vdash \alpha$. 0 ;

2) whenever $\Gamma, b: S \vdash \alpha$. 0 , we also have $\Gamma, b: T \vdash \alpha$. 0 .

Then $S$ and $T$ are interchangeable in all typing judgements.

Proof Sketch: For all $\Delta$ we prove that $\Delta, x: T \vdash P$ iff $\Delta, x: S \vdash P$. Let $x_{1}, x_{2}, a_{1}$ and $a_{2}$ be fresh names.

$$
\Delta_{i} \stackrel{\text { def }}{=} \Delta, x_{i}: T, x_{3-i}: S
$$

We will prove that $\Delta_{i} \vdash P\left\{x_{1} / x\right\}$ implies $\Delta_{i} \vdash P\left\{x_{2} / x\right\}$ for all $i \in\{1,2\}$. From there it is enough to conclude using weakening, strengthening and injective substitutions. We use $D=\overline{a_{1}} x_{1}\left|\overline{a_{2}} x_{2}\right| a_{1} y \mid a_{2} y$ to simulate a substitution:

$$
\left(\boldsymbol{\nu} x_{1} y\right)\left(D \mid P\left\{x_{1} / x\right\}\right) \Rightarrow P\left\{x_{2} / x\right\}
$$

We have to prove that $\Delta^{\prime}=\Delta_{i}, a_{1}: T_{a_{1}}, a_{2}: T_{a_{2}}, y: T_{y} \vdash D$ for some types $T_{a_{1}} T_{a_{2}}, T_{y}$. We note $a$ the subject of $\alpha$. Using the plainness of the subtyping, we can suppose that $a$ is any of $a_{1}$ or $a_{2}$ and that $b$ is any of $x_{1}, x_{2}$ or $y$, so to apply the hypothesis on different cases. There are eight subcases, along the cases from the hypothesis, $i$, and the form of $\alpha$.

- (1), $i=1, \alpha=\overline{a_{2}} x_{2}: T_{a_{1}}=T_{a_{2}}=\sharp T, T_{y}=T$;

- (1), $i=1, \alpha=a_{1} y: T_{a_{1}}=\sharp T, T_{a_{2}}=\sharp S, T_{y}=S$;

- (2), $i=1, \alpha=\overline{a_{1}} x_{1}: T_{a_{1}}=T_{a_{2}}=\sharp S, T_{y}=S$;

- (2), $i=1, \alpha=a_{2} y: T_{a_{1}}=\sharp T, T_{a_{2}}=\sharp S, T_{y}=T$;

- (1), $i=2, \alpha=\overline{a_{2}} x_{2}: T_{a_{1}}=T_{a_{2}}=\sharp T, T_{y}=T$;

- (1), $i=2, \alpha=a_{2} y: T_{a_{1}}=\sharp S, T_{a_{2}}=\sharp T, T_{y}=S$;

- (2), $i=2, \alpha=\overline{a_{1}} x_{1}: T_{a_{1}}=T_{a_{2}}=\sharp S, T_{y}=S$; 
- (2), $i=2, \alpha=a_{1} y: T_{a_{1}}=\sharp S, T_{a_{2}}=\sharp T, T_{y}=T$.

In all these cases we prove that $\Delta^{\prime} \vdash D$ using plainness and the hypothesis on $\alpha$. Plainness also give us $\Delta^{\prime} \vdash P\left\{x_{1} / x\right\}$. We use rules from (3) and Subject Reduction to get that $\Delta^{\prime} \vdash$ $P\left\{x_{2} / x\right\}$ from which strengthening is enough to conclude.

\section{Structural congruence in $\pi \mathrm{P}$ (Section $I V-A)$}

Definition 29 (Structural congruence). Structural congruence on $\pi \mathrm{P}$, written $\equiv$, is the smallest congruence containing the associativity and commutativity of $\mid$ and the following rules:

$$
\begin{gathered}
P \mid \mathbf{0} \equiv P \quad \boldsymbol{\nu} a \mathbf{0} \equiv \mathbf{0} \quad \boldsymbol{\nu} a \boldsymbol{\nu} b P \equiv \boldsymbol{\nu} b \boldsymbol{\nu} a P \\
\boldsymbol{\nu} a(P \mid Q) \equiv(\boldsymbol{\nu} a P) \mid Q \quad \text { if } a \notin \mathrm{fn}(Q)
\end{gathered}
$$

\section{Alternative definition of $\curlyvee($ Section $V$-A)}

Given an active context $E$, the set of captured names of $E$, $\operatorname{cn}(E)$, is defined as follows: $c \in \operatorname{cn}(E)$ iff the hole occurs in the scope of a restriction on $c$ in $E(\operatorname{cn}(E)$ is included in the set of names that are bound in $E$, but might be distinct from it).

Definition 30 (Reachability / Joinability of names). We introduce $\varphi::=a \leqslant b \mid a \curlyvee b$ in which $a \leqslant b$ is read " $b$ is reachable from $a$ ", and $a \curlyvee b$ is read " $a$ and $b$ are joinable". In both cases, we have $\mathrm{n}(\varphi)=\{a, b\}$. We first define a judgement $\varphi_{1}, \varphi_{2} \vdash \varphi$, as follows:

$$
\overline{a \leqslant b, b \leqslant c \vdash a \leqslant c} \quad \overline{a \leqslant c, b \leqslant c \vdash a \curlyvee b}
$$

$\overline{a \curlyvee b, c \leqslant a \vdash c \curlyvee b} \quad \overline{a \curlyvee b, c \leqslant b \vdash a \curlyvee c} \quad \frac{\varphi_{1}, \varphi_{2} \vdash \varphi}{\varphi_{2}, \varphi_{1} \vdash \varphi}$

We exploit this judgement to define how $a \leqslant b$ and $a \curlyvee b$ can be derived according to a process, or to an active context (we use $A::=P \mid E)$ :

$$
\begin{aligned}
& \text { REFL } \\
& \overline{A \triangleright a \leqslant a} \quad \frac{\begin{array}{c}
\text { DEDUCT } \\
A \triangleright \varphi_{1}
\end{array} \quad A \triangleright \varphi_{2}}{A \triangleright \varphi} \varphi_{1}, \varphi_{2} \vdash \varphi
\end{aligned} .
$$

Then we define $\triangleright$ for processes:

$$
\begin{array}{ccc}
\overline{b / a \triangleright a \leqslant b} & \frac{P \triangleright \varphi}{P \mid R \triangleright \varphi} & \frac{P \triangleright \varphi}{R \mid P \triangleright \varphi} \\
& \frac{P \triangleright \varphi \quad a \notin \mathrm{n}(\varphi)}{(\boldsymbol{\nu} a) P \triangleright \varphi} &
\end{array}
$$

and for contexts (symmetrically for $E \mid P$ ):

$$
\frac{P \triangleright \varphi \quad \mathrm{n}(\varphi) \cap \operatorname{cn}(E)=\emptyset}{P \mid E \triangleright \varphi} \quad \frac{E \triangleright \varphi}{P \mid E \triangleright \varphi} \quad \frac{E \triangleright \varphi}{(\boldsymbol{\nu} a) E \triangleright \varphi}
$$

Lemma 31. If $P$ is a $\pi \mathrm{P}$ process, the relation $\leqslant_{P}$ defined by $\{(a, b) \mid P \triangleright a \leqslant b\}$ is a preorder.

Proof: Thanks to the rule REFL, $\leqslant_{P}$ is reflexive and thanks to the rule DEDUCT and the fact that $a \leqslant b, b \leqslant c \vdash$ $a \leqslant c, \leqslant_{P}$ is transitive, hence it is a preorder.
E. Coincidence of eager and by-need equivalences in $\pi \mathrm{P} 1$ (Section VII)

Statement of Theorem 24: $\approx_{\pi \mathrm{P} 1 \mathrm{bn}}=\approx_{\pi \mathrm{P} 1 \mathrm{ea}}$.

Proof Sketch: The result follows from reflexivity of a relation we define below, between processes in the eager semantics and processes in the by-need semantics.

Lemma 32. For $P \in \pi \mathrm{P} 1$, we write $E q(P)$ for the relation between names defined by $\operatorname{Eq}(P)(a, b)$ iff $P \triangleright a \curlyvee b$.

Then $E q(P)$ is an equivalence relation.

Let $\mathcal{R}$ be the relation such that $P \mathcal{R} Q$ iff

$$
P, Q \in \pi \mathrm{P} 1 \wedge E q(P)=E q(Q)=\varphi \wedge P={ }_{\varphi} Q
$$

where $P={ }_{\varphi} Q$ iff $P$ is obtained from $Q$ by replacing some subjects in active prefixes with names related by $E q(P)$.

We prove that $P \mathcal{R} Q$ entails the following:

1) if $C[P], C[Q] \in \pi \mathrm{P} 1$ then $C[P] \mathcal{R} C[Q]$,

2) $P \Downarrow_{a}^{\text {ea }}$ iff $Q \Downarrow_{a}^{\text {bn }}$,

3) if $P \Longrightarrow$ ea $P^{\prime}$ then $Q \Longrightarrow_{\text {bn }} Q^{\prime}$ with $P^{\prime} \mathcal{R} Q^{\prime}$,

4) if $Q \Longrightarrow_{\text {bn }} Q^{\prime}$ then $P \Longrightarrow_{\text {ea }} P^{\prime}$ with $P^{\prime} \mathcal{R} Q^{\prime}$.

We call the union of relations satisfying these properties the eager/by-need weak reduction-closed barbed congruence for $\pi \mathrm{P} 1$, written ${ }_{1}^{\mathrm{ea}} \approx_{1}^{\mathrm{bn}}$.

1) $\mathcal{R}$ is clearly context-closed in $\pi \mathrm{P} 1$.

2) $P \downarrow_{a}^{\text {bn }}$ implies $P \Downarrow_{a}^{\text {ea }}$ as each arc involved in the joinability condition generates a $\longrightarrow$ ea reduction, and $P \downarrow_{a}^{\text {ea }}$ implies $P \downarrow_{a}^{\text {bn }}$, as $P \longrightarrow$ ea $P^{\prime}$ implies $P \longrightarrow_{\text {bn }} P^{\prime}$.

3) By induction we suppose $P \longrightarrow$ ea $P^{\prime}$. If this is a renaming then $P={ }_{\varphi} P^{\prime}$. If this is a communication then the corresponding subjects are equated by $\varphi$ in $Q$, which means they are joinable i.e. the by need reduction is possible.

4) Again we suppose $Q \longrightarrow_{\text {bn }} Q^{\prime}$, with a communication on $a$ and $b$ with $a \curlyvee b$. The corresponding names $a^{\prime}, b^{\prime}$ in $P$ are such that $a^{\prime} \curlyvee a \curlyvee b \curlyvee b^{\prime}$ i.e. $a^{\prime} \curlyvee b^{\prime}$ so $a^{\prime}$ and $b^{\prime}$ can be rewritten into a common name, letting the communication happen.

Since $\mathcal{R} \subseteq \underset{1}{\text { ea }} \approx_{1}^{\text {bn }}$, for all $P \in \pi \mathrm{P} 1$ we have $P{ }_{1}^{\text {ea }}{ }_{1}^{\text {bn }} P$ which implies that $P \approx_{\pi \mathrm{P} 1 \mathrm{bn}} Q$ iff $P \approx_{\pi \mathrm{P} 1 \mathrm{ea}} Q$.

F. The Fusion calculus

Definition 33. The syntax of the polyadic Fusion calculus [6] without matching and choice is the following. Structural congruence is defined as usual (Definition 29).

$$
P::=0|P| P|\bar{a} \widetilde{x} . P| a \widetilde{x} . P \mid \boldsymbol{\nu} a P .
$$

We follow the reduction semantics of the Fusion calculus, from [22]. The side conditions for (5) are that $\widetilde{x}$ and $\widetilde{y}$ are of the same arity, that $\operatorname{dom}(\sigma)=\widetilde{z}$ and that $\sigma\left(x_{i}\right)=\sigma\left(y_{i}\right)$. Note that (2), from Section II, holds.

$$
\begin{gathered}
\frac{P \equiv P_{1} \quad P_{1} \rightarrow_{F} Q_{1} \quad Q_{1} \equiv Q}{P \rightarrow_{F} Q} \quad \frac{P \rightarrow_{F} Q}{E[P] \rightarrow_{F} E[Q]} \\
(\boldsymbol{\nu} \widetilde{z})(R|a \tilde{x} . P| \bar{a} \widetilde{y} \cdot Q) \rightarrow_{F}(R|P| Q) \sigma
\end{gathered}
$$




\section{G. Auxiliary results}

a) Results involving name preorders:

Lemma 34. If $P \triangleright a \curlyvee b$ and $\{a, b\} \subseteq \mathrm{fn}(P)$, then $P \equiv P^{\prime}$ implies $P^{\prime} \triangleright a \curlyvee b$.

Proof: The predicate $P \triangleright \varphi$ only depends on the occurrences of $\operatorname{arcs}$ in $P$; those occurrences are trivially preserved by structural congruence, except that to keep track of alphaconversion one must consider that $P$ 's binders also bind $\varphi$ 's names. Hence the statement only holds for free names.

Lemma 35. If $P \simeq_{\mathrm{bn}} Q$ and $P \triangleright a \curlyvee b$. Then $Q \triangleright a \curlyvee b$.

Proof: We characterise joinability using the context $E=$ $(-|\bar{a} . f| b . g)$ where $f$ and $g$ are fresh: we easily prove that $R \triangleright a \curlyvee b$ iff $E[R] \longrightarrow_{\mathrm{bn}} R_{1}$ where $R_{1} \downarrow_{f}^{\mathrm{bn}}$ and $R_{1} \downarrow_{g}^{\mathrm{bn}}$. By definition of $\simeq_{\text {bn }}$ we know that $E[P] \simeq_{\mathrm{bn}} E[Q]$ and we conclude playing the bisimulation game of $\simeq_{\mathrm{bn}}$.

Lemma 36. If $P \mathcal{R} Q$ and $\mathcal{R}$ preserves $\curlyvee$ and parallel composition of arcs (in particular if $\mathcal{R}$ is $a \sim_{\mathrm{bn}}$-relation), then $P \triangleright a \leqslant b$ iff $Q \triangleright a \leqslant b$.

Proof: Let $P$ and $Q$ be processes and $f$ be a fresh name. Then $P \triangleright a \leqslant b$ iff $(P \mid f / b) \triangleright a \curlyvee f$ and similarly for $Q$. Thanks to the second hypothesis on $\mathcal{R}$ we have $(P \mid f / b) \mathcal{R}(Q \mid f / b)$ and we conclude with the second one.

b) Basic tools: Prefixes delimit the action of structural congruence.

Lemma 37. Suppose $\pi_{1}, \pi_{2}$ are prefixes.

1) If $E\left[\pi_{1} \cdot P_{1}\right] \equiv P^{\prime}$ then there exist $E^{\prime}$ and $P_{1}^{\prime}$ such that $P_{1} \equiv P_{1}^{\prime}, P^{\prime}=E^{\prime}\left[\pi_{1} . P_{1}^{\prime}\right]$ and $E \triangleright a \curlyvee b$ iff $E^{\prime} \triangleright a \curlyvee b$. Moreover for all $Q_{1}$ such that all names of $\mathrm{fn}\left(Q_{1}\right)$ are either in $\mathrm{fn}\left(P_{1}\right)$ or not captured by $E$ then the latter are not captured by $E^{\prime}$ and $E\left[Q_{1}\right] \equiv E^{\prime}\left[Q_{1}\right]$.

2) If $G\left[\pi_{1} \cdot P_{1}\right]\left[\pi_{2} \cdot P_{2}\right] \equiv P^{\prime}$ then there exist $G^{\prime}, P_{1}^{\prime}$ and $P_{2}^{\prime}$ such that $P_{1} \equiv P_{1}^{\prime}, P_{2} \equiv P_{2}^{\prime}$ and $P^{\prime}=$ $G^{\prime}\left[\pi_{1} . P_{1}\right]\left[\pi_{2} . P_{2}\right]$ or $P^{\prime}=G^{\prime}\left[\pi_{2} \cdot P_{2}^{\prime}\right]\left[\pi_{1} . P_{1}^{\prime}\right]$ and $G \triangleright$ $a \curlyvee b$ iff $G^{\prime} \triangleright a \curlyvee b$.

Proof: Structural congruence can act under prefixes only using the fact that $\equiv$ is a congruence, i.e. using the rule "if $P \equiv P^{\prime}$ then $C[P] \equiv C\left[P^{\prime}\right]$ " for some arbitrary context $C$ containing a prefix. For this rule we work an induction on $C$ to get the same cutting as $E\left[\pi_{1} . P_{1}\right]$; all the other rules deriving $\equiv$ are handled by the corresponding case analysis on the context $E$. Note that the statement also holds when $E$ is an arbitrary context.

Lemma 38. If $P \equiv Q$ then $P \sim_{\mathrm{bn}} Q$.

Proof: We show that $\equiv$ is a $\sim_{\mathrm{bn}}$-bisimulation. (The proof is not by induction over the derivation of $P \equiv Q$ because the fact that $\equiv$ is a congruence is not easy to handle.) The clauses $1), 2), 4$ ) are easy - respectively handled by the fact that $\equiv$ is a congruence, Lemma 34 and the fact that $\equiv$ is symmetric - as is the clause 3 ) when $\mu=\tau-$ since $\stackrel{\tau}{\longrightarrow}$ bn $=\longrightarrow_{\text {bn }}$ is stable by $\equiv$. For the remaining labels we examine the case where $\mu=b d$, the other case being similar. We know that $P=E\left[\right.$ ac. $\left.P_{1}\right]$ with $E \triangleright a \curlyvee b$ and $P^{\prime}=E\left[d / c \mid P_{1}\right]$. We use Lemma 37 to get $Q=E^{\prime}\left[a c . P_{1}\right]$ which implies $Q \stackrel{b d}{\longrightarrow} E^{\prime}\left[d / c \mid P_{1}\right] \equiv P^{\prime}$.

\section{c) Proof techniques:}

Definition 39 (By-need bisimulation up to $\sim_{\text {bn }}$ and restriction). A relation $\mathcal{R}$ is a by-need bisimulation up to $\sim_{\text {bn }}$ and restriction if $P \mathcal{R} Q$ implies:

1) $P|a / b \mathcal{R} Q| a / b$, for all names $a, b$;

2) if $a$ and $b$ appear free in $P$, then $P \triangleright a \curlyvee b$ implies $Q \triangleright a \curlyvee b$;

3) if $P \stackrel{\mu}{\longrightarrow}$ bn $P^{\prime}$ (where the object part of $\mu$ is fresh, whenever $\mu \neq \tau$ ), then $Q \stackrel{\mu}{\longrightarrow}$ bn $Q^{\prime}$ and there are $P^{\prime \prime}, Q^{\prime \prime}, \widetilde{x}$ s.t. $P^{\prime} \sim_{\text {bn }} \nu \widetilde{x} P^{\prime \prime}, Q^{\prime} \sim_{\text {bn }} \nu \widetilde{x} Q^{\prime \prime}$, and $P^{\prime \prime} \mathcal{R} Q^{\prime \prime}$,

4) the converse of clauses (2) and (3).

Lemma 40. If $\mathcal{R}$ is a by-need bisimulation up to $\sim_{\mathrm{bn}}$ and restriction then $\mathcal{R} \subseteq \sim_{\text {bn }}$.

\section{H. Soundness of $\sim_{\mathrm{bn}}($ Section $V$-C)}

We now move to the proof that $\sim_{b n}$ is a congruence. What is missing is closure by parallel composition, which is rather delicate. This is because we defined the semantics of $\tau$-actions with a reduction semantics. (The standard schema is to define a pure SOS semantics, show that it coincides with the reduction semantics, and then work with the SOS.)

For the proof of congruence we introduce communication contexts. These are, intuitively, the composition of two active contexts, one used for an input, the other for an output; such input and output may produce a $\tau$-action. Communication contexts, ranged over by $G$, have two holes, each occurring exactly once.

$$
G::=P|G| G|P| \boldsymbol{\nu} a G\left|E_{1}\right| E_{2} .
$$

By convention the leftmost hole is the first one, the other is the second one. We write $P=G[a c . Q][\bar{b} d . R]$ if $P$ is obtained from $G$ with $a c . Q$, and the second hole with $\bar{b} d$. $R$.

Communication contexts can be used to decompose a $\stackrel{\tau}{\longrightarrow}$ bn transition:

Lemma 41. Suppose $P \stackrel{\tau}{\longrightarrow}_{\text {bn }} P^{\prime}$ (that is, $P \longrightarrow_{\mathrm{bn}} P^{\prime}$ ). Then one of the following statements holds:

- $P=G[\bar{a} b . Q][c d . R]$ and $P^{\prime} \sim_{\mathrm{bn}} \boldsymbol{\nu} f(G[b / f \mid Q][f / d \mid R])$, - $P=G[c d . R][\bar{a} b . Q]$ and $P^{\prime} \sim_{\mathrm{bn}} \boldsymbol{\nu} f(G[f / d \mid R][b / f \mid Q])$, where $P \triangleright a \curlyvee c$ and $f$ is fresh.

Proof: The two cases are similar, the main difficulty is to keep track of the structural congruence operations. If $P \longrightarrow$ bn $P^{\prime}$ it means that, $P \equiv E\left[\bar{a} b . Q_{1} \mid a c . R_{1}\right]$ and $P^{\prime} \equiv E[b / c \mid$ $Q_{1} \mid R_{1}$. From the first relation we can get $G$ such that $P=G[\bar{a} b . Q][c d . R]$ (with $G \triangleright a \curlyvee c, Q \equiv Q_{1}$ and $R \equiv R_{1}$ ), ignoring the symmetric case for which the output is the left argument of $G$. We extract the potential restrictions $\boldsymbol{\nu} \hat{b}$ and $\boldsymbol{\nu} \hat{d}$ $(\hat{b}=\emptyset$ if $b$ is not bound and $\hat{b}=\{b\}$ if is captured by $G$ ) from $G$, yielding the much alike context $G^{\prime}$ (and $G \equiv(\nu \hat{b} \hat{d}) G^{\prime}$ ). 
The interesting part is that we can write the reduction with the arc at the top, then use Lemma 44 and then structural congruence to put back $b$ and $d$ inside $G$.

$$
\begin{aligned}
& P \equiv(\boldsymbol{\nu} \hat{b} \hat{d}) G^{\prime}[\bar{a} b . Q][c d . R] \\
& \longrightarrow_{\text {bn }}(\boldsymbol{\nu} \hat{b} \hat{d})\left(b / d \mid G^{\prime}[Q][R]\right) \\
& \sim_{\text {bn }}(\boldsymbol{\nu} \hat{b} \hat{d})\left((\boldsymbol{\nu} f)(b / f \mid f / d) \mid G^{\prime}[Q][R]\right) \\
& \quad \equiv(\boldsymbol{\nu} f)(\boldsymbol{\nu} \hat{b} \hat{d})\left(G^{\prime}[b / f \mid Q][f / d \mid R]\right) \\
& \quad \equiv(\boldsymbol{\nu} f) G[b / f \mid Q][f / d \mid R] .
\end{aligned}
$$

To conclude we need to relate this last process to $P^{\prime}$ which is done by proving that $E\left[b / d\left|Q_{1}\right| R_{1}\right] \equiv(\boldsymbol{\nu} \hat{b} \hat{d})(b / d \mid$ $G^{\prime}[Q][R]$ ), which is done by keeping tracks of the derivation of $E\left[\bar{a} b . Q_{1} \mid c d . R_{1}\right] \equiv P$.

Lemma 42. Suppose $Q \stackrel{b f}{\rightarrow}$ bn $Q^{\prime}$ and suppose $b$ is not captured by $E$. Then $Q \mid E\left[\bar{b} d . R_{1}\right] \stackrel{\tau}{\longrightarrow}{ }_{\mathrm{bn}} \sim_{\mathrm{bn}} \boldsymbol{\nu} f\left(E\left[d / f \mid R_{1}\right]\right)$.

Lemma 43 (Congruence for restriction). If $P \sim_{\mathrm{bn}} Q$ then for all $c, \boldsymbol{\nu} c P \sim_{\mathrm{bn}} \boldsymbol{\nu} c Q$.

Proof: Given a relation $\mathcal{R}$, we define

$$
(\mathcal{R})^{\mathrm{Sub}}=\{(P|\sigma, Q| \sigma) \cdot P \mathcal{R} Q \text { and } \sigma \text { is }
$$
a parallel composition of arcs $\}$.

We show that $\left(\left\{(\boldsymbol{\nu} c P, \boldsymbol{\nu} c Q), P \sim_{\mathrm{bn}} Q\right\}\right)^{\mathrm{Sub}}$ is a bisimulation up to $\equiv$. This is a consequence of the following observations:

- For any $u, v, c, P$ such that $\{u, v\} \subseteq \mathrm{fn}(P)$ and $c \notin$ $\{u, v\}$, we have $P \triangleright u \curlyvee v$ iff $\boldsymbol{\nu} c P \triangleright u \curlyvee v$.

- The visible transitions of our labelled transition system do not involve name extrusion, and we have that $P \stackrel{\alpha}{\longrightarrow}$ bn $P^{\prime}$ iff $\boldsymbol{\nu} c P \stackrel{\alpha}{\longrightarrow}$ bn $\boldsymbol{\nu} c P^{\prime}$ for $c \notin \mathrm{n}(\alpha)$.

- Suppose now $\boldsymbol{\nu} c P \stackrel{\tau}{\longrightarrow}$ bn $P^{\prime}$. This means $P \stackrel{\tau}{\longrightarrow}$ bn $P_{0}$ for some $P_{0}$ s.t. $P^{\prime} \equiv \nu c P_{0}$. But then $Q \stackrel{\tau}{\longrightarrow}$ bn $Q_{0}$, $P_{0} \sim_{\text {bn }} Q_{0}$ and $\boldsymbol{\nu} c Q \stackrel{\tau}{\longrightarrow}$ bn $\nu c Q_{0}$.

Lemma 44 (Transitivity of arcs). For all active context $E$ we have: $E[a / c] \sim_{\text {bn }} E[\boldsymbol{\nu} b(a / b \mid b / c)]$.

Proof: Let $\mathcal{R}$ be the corresponding relation. We show that $\mathcal{R}$ is a $\sim_{\text {bn }}$-bisimulation up to $\equiv$. Of course the relation is stable by parallel composition of arcs, since $E$ can be an arbitrary active context. Concerning the $\curlyvee$ condition, the leftto-right implication is rather clear. From right to left, we must prove that we cannot get more from $\nu b(a / b \mid b / c)$ than from $a / c$ which is achieved by the restriction $\nu b$. Now concerning the transitions we know from the $\checkmark$ condition that the same names will be joinable through the preorder, independently of $\equiv$ or the context. The resulting processes will still stay in $\mathcal{R}$, up to $\equiv$.

Lemma 45 (Congruence for parallel composition). If $P \sim_{\mathrm{bn}}$ $Q$ then also $P\left|R \sim_{\text {bn }} Q\right| R$.

Proof (Sketch): Special case: we first suppose that all arcs in $R$ occur under at least one prefix. We show that $\left\{(P|R, Q| R), P \sim_{\text {bn }} Q\right.$ and $R$ does not contain active arcs $\}$ by contexts. is a bisimulation up to restriction and up to bisimilarity.

Suppose then $P \mid R \stackrel{\tau}{\longrightarrow}$ bn $U$, in which both $P$ and $R$ contribute (the other possibilities are easier).

Suppose $P$ makes the input (the case of output is symmetric). In this case we have, using Lemma 41:

$$
P=E\left[\text { ac. } P_{1}\right] \quad R=F\left[\bar{b} d . R_{1}\right]
$$

where $E \triangleright a \curlyvee b$ (since $R$ has no arc) and with $P^{\prime}=E\left[f / c \mid P_{1}\right]$ and $R^{\prime}=F\left[d / f \mid R_{1}\right]$ :

$$
U \sim_{\mathrm{bn}} \boldsymbol{\nu} f\left(P^{\prime} \mid R^{\prime}\right)
$$

Using rule EN-INP, we also have $P \stackrel{b f}{\longrightarrow}_{\text {bn }} P^{\prime}$. Hence, since $P \sim_{\text {bn }} Q, Q \stackrel{b f}{\rightarrow}_{\text {bn }} Q^{\prime}$ and $P^{\prime} \sim_{\text {bn }} Q^{\prime}$ for some $Q^{\prime}$, which gives $Q^{\prime}=E^{\prime}\left[a^{\prime} c^{\prime} . Q_{1}\right]$ for some $a^{\prime}$ s.t. $E^{\prime} \triangleright a^{\prime} \curlyvee b$, and $Q^{\prime}=E^{\prime}\left[f / c^{\prime} \mid Q_{1}\right]$. From this, Lemma 42 gives us directly:

$$
Q \mid R \stackrel{\tau}{\longrightarrow}_{\mathrm{bn}} \sim_{\mathrm{bn}} \nu f\left(Q^{\prime} \mid R^{\prime}\right)
$$

We can now extract the arc from $R^{\prime}$ :

$$
R^{\prime} \equiv \boldsymbol{\nu} \widetilde{n}\left(R^{\prime \prime} \mid \sigma\right)
$$

where $\sigma$ is a parallel composition of arcs and $R^{\prime \prime}$ contains no active arc. We then have

$$
P^{\prime} \mid R^{\prime} \equiv(\boldsymbol{\nu} \tilde{n})\left(P^{\prime}|\sigma| R^{\prime \prime}\right),
$$

and similarly for $Q^{\prime} \mid R^{\prime}$. We can conclude by remarking that $P^{\prime} \sim_{\text {bn }} Q^{\prime}$ entails $P^{\prime}\left|\sigma \sim_{\text {bn }} Q^{\prime}\right| \sigma$, and using up to restriction to remove the topmost restrictions.

General case: Consider now the case where $R$ is an arbitrary process. We reason by induction on $R$, to show that $P \sim_{\text {bn }} Q$ implies $P\left|R \sim_{\text {bn }} Q\right| R$. The cases where $R$ is a prefixed process or $R=\mathbf{0}$ are treated by the result above.

The case where $R=u / v$ holds by definition of $\sim_{\mathrm{bn}}: P \sim_{\mathrm{bn}}$ $Q$ implies $P\left|u / v \sim_{\text {bn }} Q\right| u / v$.

If $R=R_{1} \mid R_{2}$, then by induction $P\left|R_{1} \sim_{\mathrm{bn}} Q\right| R_{1}$, which gives, by induction again, $\left(P \mid R_{1}\right) \mid R_{2} \sim_{\mathrm{bn}}(Q \mid$ $\left.R_{1}\right) \mid R_{2}$, hence the result by associativity of $\mid$.

Suppose now $R=\boldsymbol{\nu} c R^{\prime}$. We can suppose w.l.o.g. $c \notin$ $\mathrm{fn}(P) \cup \mathrm{fn}(Q)$. Then by induction $P\left|R^{\prime} \sim_{\mathrm{bn}} Q\right| R^{\prime}$, which gives, by Lemma 43, $(\boldsymbol{\nu} c)\left(P \mid R^{\prime}\right) \sim_{\mathrm{bn}}(\boldsymbol{\nu} c)\left(Q \mid R^{\prime}\right)$. Lemma 38 gives $(\boldsymbol{\nu} c)\left(P \mid R^{\prime}\right) \sim_{\text {bn }} P \mid \boldsymbol{\nu} c R^{\prime}$, and similarly for $Q$, hence $P \mid R \sim$ bn $Q \mid R$. This concludes the proof.

\section{Statement of Theorem 20: Bisimilarity is a congruence.}

Proof: Follows from Lemmas 43 and 45, closure by prefixes being immediate.

Theorem 46 (Soundness). If $P \sim_{\mathrm{bn}} Q$ then $P \simeq_{\mathrm{bn}} Q$.

Proof: Preservation of fresh barbs: when $f$ does not appear in any arc, $P \downarrow_{f}^{\text {bn }}$ is equivalent to $P \stackrel{\alpha}{\longrightarrow}$ where $\alpha$ is an input or output label with subject $f$.

Preservation of general barbs: $P \downarrow_{a}^{\mathrm{bn}}$ is equivalent to $(P \mid$ $\alpha . f) \stackrel{\tau}{\longrightarrow}$ bn $\downarrow_{f}^{\text {bn }}$ for some $\alpha$ whose subject is $a$.

Closure under reduction holds trivially since $\longrightarrow_{\text {bn }}$ coincides with $\stackrel{\tau}{\longrightarrow}$ bn and finally, Theorem 20 guarantees closure 


\section{Completeness of $\sim_{\mathrm{bn}}($ Section V-C)}

For a prefix $\alpha$ we write $\bar{\alpha}$ for the dual prefix, i.e. $\overline{\bar{a}} \bar{b}=a b$ and $\overline{a b}=\bar{a} b$. Any prefix $\alpha$ can be also seen as a label.

Lemma 47. Let $P$ and $P^{\prime}$ be processes and $f$ a name fresh w.r.t. $P$ and such that $P^{\prime} \downarrow_{f}^{\mathrm{bn}}$. Then $P \stackrel{\alpha}{\longrightarrow}{ }_{\mathrm{bn}} \equiv P^{\prime}$ if and only if there exists a process $P_{1}$ such that $P_{1} \downarrow_{f}^{\mathrm{bn}}$ and

$$
P \mid \bar{\alpha} \cdot(\bar{f} \mid f) \longrightarrow_{\text {bn }} P_{1} \longrightarrow_{\text {bn }} P^{\prime} .
$$

Proof: Let us consider the case where $\alpha$ is an input prefix $b d$, the output case being similar.

Left to right: since $\longrightarrow_{\text {bn }}$ is stable by $\equiv$ we directly suppose that $P \stackrel{\alpha}{\longrightarrow}$ bn $P^{\prime}$. Then $P=E[a c . Q]$ with $E \triangleright a \curlyvee b$ and $P^{\prime}=E[d / c \mid Q]$. Then

$$
\begin{aligned}
P_{\alpha} & \stackrel{\text { def }}{=} P \mid \bar{\alpha} \cdot(\bar{f} \mid f) \\
& \equiv E[a c \cdot Q \mid \bar{b} d \cdot(\bar{f} \mid f)] \\
& \longrightarrow \text { bn } E[d / c|Q| \bar{f} \mid f] \stackrel{\text { def }}{=} P_{1} \\
& \longrightarrow \text { bn } E[d / c \mid Q]=P^{\prime} .
\end{aligned}
$$

Right to left: since $P_{1}$ and $f$ is fresh in $P$ we know that $\bar{\alpha}$ has been triggered, that is, $P_{\alpha} \equiv E[a c . Q \mid \bar{b} d .(\bar{f} \mid f)]$ and $P^{\prime} \equiv E[d / c \mid Q]$ since $P^{\prime}$ has no $f$ barb. This means that $P$ is of the form $P \equiv E[a c . Q]$. Hence $P \stackrel{\alpha}{\longrightarrow}{ }_{\mathrm{bn}} \equiv P^{\prime}$.

Theorem 48 (Completeness). If $P \simeq_{\mathrm{bn}} Q$ then $P \sim_{\mathrm{bn}} Q$.

Proof: We show that $\simeq_{\text {bn }}$ is a $\sim_{\text {bn }}$-bisimulation up to $\equiv$. The clause for preservation of $\gamma$ is treated with Lemma 35 . The one about parallel composition of arcs is trivial, as well as the symmetry and the clause for the $\tau$-transition. We are left with the case for an input or output transition $\alpha$.

Suppose $P \stackrel{\alpha}{\longrightarrow}$ bn $P^{\prime}$ and let $f$ be a name fresh wrt to $P$, $P^{\prime}$ and $Q$. Lemma 47 provides us $P_{1}$ such that $P_{1} \downarrow_{f}^{\text {bn }}$ and a reduction scheme that we can transport to $Q$ :

$$
Q \mid \bar{\alpha} \cdot(\bar{f} \mid f) \longrightarrow_{\text {bn }} Q_{1} \longrightarrow_{\text {bn }} Q_{2} .
$$

We know that $P_{1} \simeq_{\text {bn }} Q_{1}$ and $P^{\prime} \simeq_{\text {bn }} Q_{2}$, hence $Q_{1} \downarrow_{f}^{\text {bn }}$ and $Q_{2} \downarrow_{f}^{\text {bn }}$ (since $f$ is fresh for $P^{\prime}$ ). Another application of Lemma 47 directly gives us $Q \stackrel{\alpha}{\longrightarrow}$ bn $\equiv Q_{2}$.

Statement of Theorem 21: In $\pi \mathrm{P}$, relations $\sim_{\mathrm{bn}}$ and $\simeq_{\mathrm{bn}}$ coincide.

Proof: Consequence of Theorems 48 and 46.

\section{J. Encoding $\mathrm{A} \pi$ in $\pi \mathrm{P}$}

1) Operational correspondence results: We say that $P \in$ $\pi \mathrm{P}$ is asynchronous if the continuation of all outputs in $P$ is 0 . We can remark that the encoding of a process in $\mathrm{A} \pi$ is an asynchronous $\pi \mathrm{P}$ process.

We use the following properties of the encoding, where $\longrightarrow_{\pi}$ is the reduction in the $\pi$-calculus. Barbs in the $\pi$-calculus are defined in the standard way: $P \downarrow_{a}$ iff $P \equiv(\boldsymbol{\nu} \widetilde{c})(\alpha . P \mid R)$ where $\alpha$ is a prefix whose subject is $a$. (It is equivalent to $P=E\left[\alpha . P_{1}\right]$ for some active context $E$.)

Lemma 49. Let $P$ be any $\pi$-calculus process.
1) If $P \equiv Q$ then $\llbracket P \rrbracket \equiv \llbracket Q \rrbracket$;

2) if $\llbracket P \rrbracket \equiv \llbracket Q \rrbracket$ then $P \equiv Q$;

3) if $\llbracket P \rrbracket \equiv E_{1}\left[\bar{a} b . Q_{1} \mid\right.$ ax. $\left.R_{1}\right]$ then $Q_{1} \equiv \llbracket Q \rrbracket, R_{1} \equiv \llbracket R \rrbracket$ and $P \equiv E[\bar{a} b . Q \mid a(x) . R]$ with $\llbracket E \rrbracket[\boldsymbol{\nu} x[\cdot]] \equiv E_{1}[\cdot]$.

4) if $P \longrightarrow_{\pi} P^{\prime}$ then $\llbracket P \rrbracket \longrightarrow_{\mathrm{bn}} \simeq_{\mathrm{bn}} \llbracket P^{\prime} \rrbracket$;

5) conversely, if $\llbracket P \rrbracket \longrightarrow_{\mathrm{bn}} P_{1}$ then there is $P^{\prime}$ such that $P \longrightarrow_{\pi} P^{\prime}$ and $P_{1} \simeq_{\mathrm{bn}} \llbracket P^{\prime} \rrbracket ;$

6) $P \downarrow_{a}$ iff $\llbracket P \rrbracket \downarrow_{a}$.

Proof:

1) Straightforward

2) We prove tediously but straightforwardly the following refined statement: if $\llbracket P \rrbracket \equiv R_{1}$ then there exist $R$ such that $P \equiv R$ and we can obtain $R_{1}$ from $\llbracket R \rrbracket$ such that $R_{1} \equiv \llbracket R \rrbracket$ but only by moving restrictions of input objects. In the case where $R_{1}=\llbracket Q \rrbracket$ we prove that $R$ is necessarily $Q$ (the restrictions of input objects have only one possible position).

3) We combine techniques used in the previous item to get back the fact $Q_{1}$ and $R_{1}$ are structurally congruent to encoding of processes, and techniques from the proof of Lemma 37 to separate the transformations of $\equiv$ in the subterms $Q_{1}, R_{1}$ guarded by the prefixes $\bar{a} b$, ax from those in the rest of the term.

4) The reduction $\longrightarrow_{\pi}$ is quotiented by structural congruence, so in the induction proof there is a case handling the rule "if $P \equiv P_{1} \longrightarrow_{\pi} P_{1}^{\prime} \equiv P^{\prime}$ then $P \longrightarrow_{\pi} \equiv P^{\prime \prime}$. Since $\llbracket P \rrbracket \equiv \llbracket P_{1} \rrbracket$ and $\llbracket P_{1}^{\prime} \rrbracket \equiv \llbracket P^{\prime} \rrbracket$ we only need to know that $\llbracket P_{1} \rrbracket \longrightarrow_{\mathrm{bn}} \simeq_{\mathrm{bn}} \llbracket P_{1}^{\prime} \rrbracket$ by induction. We also need to know that $\left(\equiv \longrightarrow_{b_{n}} \simeq_{\text {bn }} \equiv\right) \subseteq\left(\longrightarrow_{\text {bn }} \simeq_{\text {bn }}\right)$ which is true by definition of $\longrightarrow_{\mathrm{bn}}$ and $\simeq_{\mathrm{bn}}$.

Similarly since the reduction in $\pi$ is also quotiented by active contexts we also remark that the encoding is compositional, and the encoding of an active context is still active. Also we have to prove that if $P \longrightarrow_{\mathrm{bn}} \simeq_{\mathrm{bn}} Q$ then $P \longrightarrow_{\mathrm{bn}} \simeq_{\mathrm{bn}} Q$ which is true by definition of $\longrightarrow_{\text {bn }}$ and because $\simeq_{\text {bn }}$ is a congruence.

We now focus on the simple case of $\bar{a} b . P$ $a(x) . Q \longrightarrow_{\pi} P \mid Q\{b / x\}$. The encoding of the lefthand side reduces into $\boldsymbol{\nu} x(\llbracket P \rrbracket|b / x| \llbracket Q \rrbracket)$ and we know that $x$ has no negative occurrence in $\llbracket Q \rrbracket$ so by Lemma 19 this process is equivalent to $\llbracket P \rrbracket \mid \llbracket Q \rrbracket\{b / x\}$ which is of the expected shape.

5) If $\llbracket P \rrbracket \longrightarrow_{\mathrm{bn}} Q$, since $\llbracket P \rrbracket$ does not have any arc, the reduction comes from a communication between two prefixes on the same name $a: \llbracket P \rrbracket \equiv E_{1}[\bar{a} b . \llbracket Q \rrbracket \mid$ $a x . \llbracket R \rrbracket$ with $E$ binding $x$, and then keeping track of all actions operated by $\equiv$ we know that $P_{1}$ is of the form $P_{1} \equiv E_{1}[\llbracket Q \rrbracket|b / x| \llbracket R \rrbracket]$. We can recover $P \equiv E[\bar{a} b . Q \mid a(x) . R] \longrightarrow_{\pi} E[Q \mid R\{b / x\}] \stackrel{\text { def }}{=} P^{\prime}$. Then $\llbracket P^{\prime} \rrbracket=\llbracket E \rrbracket[\llbracket Q \rrbracket \mid \llbracket R \rrbracket\{b / x\}] \equiv E_{1}[\llbracket Q \rrbracket \mid$ $\llbracket R \rrbracket\{b / x\}] \simeq_{\text {bn }} P_{1}$.

6) The implication from left to right is straightforward by induction, but one has to remark that to test the input barb, one needs a synchronous tester $\bar{a} b . \omega$. (Note that input barbs are not tested in the asynchronous version of 
behavioural equivalences.) The other implication follows from the fact that there is no arc in $\llbracket P \rrbracket$ so $\llbracket P \rrbracket \downarrow_{a}$ if and only if $\llbracket P \rrbracket$ contains a prefix whose subject is $a$ (which is equivalent to the fact $P$ does, too).

Lemma 50 (Label-syntax correspondence). If $P$ is only contains trivial arcs (of the form e/e) and $\alpha$ is a prefix ac or $\bar{a} c$ then $P \stackrel{\alpha}{\longrightarrow} \mathrm{bn}_{\mathrm{n}} \equiv P^{\prime}$ iff $P \equiv E\left[\alpha . P_{1}\right]$ and $P^{\prime} \equiv E\left[c / c \mid P_{1}\right]$, with $E$ binding neither a nor $c$ (and $P^{\prime}$ has only trivial arcs).

Moreover $P \downarrow_{a}^{\text {bn }}$ iff $P \stackrel{\alpha}{\longrightarrow}$ bn iff $P \equiv E\left[\alpha . P_{1}\right]$.

In addition if $\sigma \triangleright a \curlyvee b$ then $P \stackrel{a c}{\longrightarrow}$ bn $P^{\prime}$ implies $P \mid \sigma \stackrel{b c}{\longrightarrow}$ bn $P^{\prime} \mid \sigma($ resp. $\bar{a} c, \bar{b} c)$.

Lemma 51 (Label correspondences). Let $P$ be any $\pi$ process and $f$ a fresh name.

1) If $P \stackrel{\bar{a} c}{\longrightarrow}{ }_{\pi} P^{\prime}$ then $\llbracket P \rrbracket \stackrel{\bar{a} f}{\longrightarrow}_{\mathrm{bn}} \equiv c / f \mid \llbracket P^{\prime} \rrbracket$.

2) If $P \stackrel{\bar{a}(c)}{\longrightarrow}{ }_{\pi} P^{\prime}$ then $\llbracket P \rrbracket \stackrel{\bar{a} f}{\longrightarrow}$ bn $\equiv \boldsymbol{\nu} c\left(c / f \mid \llbracket P^{\prime} \rrbracket\right)$.

3) If $P \stackrel{a(x)}{\longrightarrow}{ }_{\pi} P^{\prime}$ then $\llbracket P \rrbracket \stackrel{a f}{\longrightarrow}$ bn $\equiv \boldsymbol{\nu} x\left(f / x \mid \llbracket P^{\prime} \rrbracket\right)$.

4) If $\llbracket P \rrbracket \stackrel{\bar{a} f}{\longrightarrow}$ bn $P_{1}$ then

a) either $P \stackrel{\bar{a} c}{\longrightarrow}_{\pi} P^{\prime}$ with $P_{1} \equiv c / f \mid \llbracket P^{\prime} \rrbracket$,

b) or $P \stackrel{\bar{a}(c)}{\longrightarrow} P^{\prime}$ with $P_{1} \equiv \boldsymbol{\nu} c\left(c / f \mid \llbracket P^{\prime} \rrbracket\right)$

5) If $\llbracket P \rrbracket \stackrel{a f}{\longrightarrow}$ bn $P_{1}$ then

$P \stackrel{a(x)}{\longrightarrow} P^{\prime}$ with $P_{1} \equiv \boldsymbol{\nu} x .\left(f / x \mid \llbracket P^{\prime} \rrbracket\right)$

Lemma 52 (Decomposition of transitions, asynchronous $\pi \mathrm{P}$ ). Let $P$ be an asynchronous $\pi \mathrm{P}$ term without visible arc, $\sigma$ a parallel composition of arcs, and $f, g$ some fresh names.

1) If $P \mid \sigma \stackrel{\tau}{\longrightarrow}$ bn $P_{t}$ then $P \stackrel{\bar{a} f}{\longrightarrow}$ bn $P_{1} \stackrel{b g}{\longrightarrow}$ bn $P_{2}$ with $P_{t} \sim_{\text {bn }}(\boldsymbol{\nu} f g)\left(P_{2} \mid f / g\right) \mid \sigma$ and $\sigma \triangleright a \curlyvee b$.

2) Suppose $P \stackrel{\bar{a} f}{\longrightarrow}$ bn $P_{1} \stackrel{a g}{\longrightarrow}$ bn $P_{2}$ and $\sigma \triangleright a \curlyvee b$. Then $P\left|\sigma \stackrel{\tau}{\longrightarrow}_{\mathrm{bn}} \sim{ }_{\mathrm{bn}}(\boldsymbol{\nu} f g)\left(P_{2} \mid f / g\right)\right| \sigma$.

This result is directly a consequence of the syntax of asynchronous $\pi \mathrm{P}$ as for similar results in $\mathrm{A} \pi$. We use $\sim_{\mathrm{bn}}$ for renaming and concatenating fresh names using Lemma 44.

2) Full abstraction for the encoding of $\mathrm{A} \pi$ : One inclusion in the full abstraction result actually holds for the whole $\pi$ calculus:

Lemma 53. Let $P$ and $Q$ be $\pi$ terms. Then $\llbracket P \rrbracket \simeq_{\mathrm{bn}} \llbracket Q \rrbracket$ implies $P \simeq_{\pi} Q$.

Proof: The relation $\left\{(P, Q) \mid \llbracket P \rrbracket \simeq_{\mathrm{bn}} \llbracket Q \rrbracket\right\}$ is reductionclosed (consequence of Lemma 49), barb-preserving (consequence of Lemma 49), and context-closed: if $C$ is a $\pi$ context then there exists a $\pi \mathrm{P}$ context $C_{1}$ such that $\left.\llbracket C[P] \rrbracket=C_{1} \llbracket \llbracket P \rrbracket\right]$, similarly for $Q$; hence $\llbracket P \rrbracket \simeq_{\mathrm{bn}} \llbracket Q \rrbracket$ implies $\llbracket C[P] \rrbracket \simeq_{\mathrm{bn}}$ $\llbracket C[Q] \rrbracket$.

Lemma 54. Let $P$ and $Q$ be asynchronous $\pi$-terms. Then $P \simeq_{\pi} Q$ implies $\llbracket P \rrbracket \simeq_{\text {bn }} \llbracket Q \rrbracket$.

Proof: Thanks to Theorem 46 and to the characterisation of barbed congruence by ground bisimilarity in the asynchronous $\pi$-calculus [5], we only have to prove that $P \sim_{\mathrm{g}} Q$ implies $\llbracket P \rrbracket \sim_{\text {bn }} \llbracket Q \rrbracket$. We do so by showing that the following relation is a $\sim_{\text {bn }}$-bisimulation up to restriction and $\sim_{\text {bn }}$ :

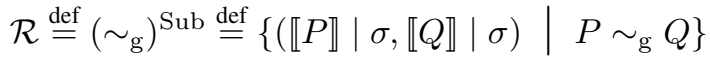

where $\sigma$ stands for any parallel composition of arcs. In order to do that, we rely on Lemma 51 ( $\llbracket P \rrbracket$ is arc-free) to relate non- $\tau$ transitions in $\pi$ and $\pi \mathrm{P}$, as well as on Lemma 52 to decompose $\tau$-transitions into visible transitions.

We analyse all possible transitions from $\llbracket P \rrbracket \mid \sigma$. We omit intermediate steps to focus on the relevant details.

1) $\llbracket P \rrbracket \mid \sigma \stackrel{a f}{\longrightarrow}_{\mathrm{bn}} \sim_{\mathrm{bn}} \boldsymbol{\nu} x\left(f / x\left|\llbracket P^{\prime} \rrbracket\right| \sigma\right)$ with $P \stackrel{b(x)}{\longrightarrow}{ }_{\pi}$ $P^{\prime}$ for some $b$ such that $\sigma \triangleright a \curlyvee b$. Drawing the $\sim_{\mathrm{g}^{-}}$ diagram yields eventually $\llbracket Q \rrbracket \stackrel{b f}{\longrightarrow}{ }_{\text {bn }} \sim_{\text {bn }} \boldsymbol{\nu} x(f / x \mid$ $\left.\llbracket Q^{\prime} \rrbracket\right)$. We add $\sigma$ to derive a transition along the original label $a f$, and relate in $\mathcal{R}$ the resulting processes.

2) $\llbracket P \rrbracket \mid \sigma \stackrel{\bar{a} f}{\longrightarrow}_{\mathrm{bn}} \sim_{\mathrm{bn}} \boldsymbol{\nu} \hat{c}\left(c / f \mid \llbracket P^{\prime} \rrbracket\right)$ with $P \stackrel{\boldsymbol{\nu} \hat{c} b c}{\longrightarrow}_{\pi} P^{\prime}$ with $\hat{c} \in\{\emptyset,\{c\}\}$ and $\sigma \triangleright a \curlyvee b$. The reasoning is similar to the previous case.

3) $\llbracket P \rrbracket \mid \sigma \stackrel{\tau}{\longrightarrow}$ bn $P_{t} \mid \sigma$ with

$$
\begin{gathered}
\llbracket P \rrbracket \stackrel{\bar{a} f}{\longrightarrow}_{\mathrm{bn}} \stackrel{b g}{\longrightarrow}_{\mathrm{bn}} \boldsymbol{\nu} \hat{c} x\left(c / f|g / x| \llbracket P^{\prime \prime} \rrbracket\right) \stackrel{\text { def }}{=} P_{2} \\
P \stackrel{\boldsymbol{\nu} \hat{c} a c}{\longrightarrow}_{\pi} \stackrel{b(x)}{\longrightarrow}_{\pi} P^{\prime \prime}
\end{gathered}
$$

such that $\sigma \triangleright a \curlyvee b$ and $P_{t} \sim_{\text {bn }} \nu f g\left(P_{2} \mid f / g\right)$. We can again play the ground bisimilarity game and use Lemma 52 to get the same relations on the $Q$ side, to finally get $P \sim_{\mathrm{g}} Q$ and thus:

$$
\left(\llbracket P^{\prime \prime} \rrbracket \mid \sigma^{\prime}\right) \mathcal{R}\left(\llbracket Q^{\prime \prime} \rrbracket \mid \sigma^{\prime}\right)
$$

with $\sigma^{\prime}=\sigma|c / f| f / g \mid g / x$. We use the up to restriction technique on $f, g, x$, and $\hat{c}$.

The relation $\mathcal{R}$ is symmetric, and clearly satisfies the clause about joinability and the clause about the addition of arcs. Thus $\mathcal{R}$ is a $\sim_{\text {bn }}$-bisimulation up to restriction and $\sim_{\text {bn }}$.

Theorem 55 (Full abstraction). Suppose $P, Q$ are processes from the asynchronous $\pi$-calculus, $\mathrm{A} \pi$. Then $P \simeq_{\mathrm{A} \pi} Q$ iff $\llbracket P \rrbracket \simeq_{\text {bn }} \llbracket Q \rrbracket$.

\section{K. Encoding of Explicit Fusions}

Definition 56. Let $P \triangleright a=b$ be the judgement conjunction of $P \triangleright a \leqslant b$ and $P \triangleright b \leqslant a$.

In the following we note $\varphi_{P}$ the relation $\{(a, b) \mid P \triangleright a \varphi b\}$, e.g. $a \curlyvee_{P} b$ for the joinability $a \leqslant_{P} b$ for the reachability or $a={ }_{P} b$ for the equality. We will note $P={ }_{a, b} Q$ iff $P\{b / a\}=$ $Q\{b / a\}$ i.e. if the only difference between $P$ and $Q$ is the exchange of some $a$ and $b$. We will also write $a=b$ for $\llbracket a=b \rrbracket$ which is $a / b \mid b / a$.

Lemma 57. If $P={ }_{a, b} Q$ then $\varphi_{P \mid a=b}=\varphi_{Q \mid a=b}$.

Proof: By symmetry we only consider inclusion. We use induction on the derivation of $(P \mid a=b) \triangleright \varphi$ along Definition 30. Only the base case is interesting, when $P$ and $Q$ are $\operatorname{arcs}$ and $\varphi$ is of the form $d \leqslant e$. Then if $\mathrm{n}(\varphi) \subseteq\{a, b\}$ 
then $(a=b) \triangleright \varphi$; if $P \neq Q$ then $(P, Q)$ can only be of the form $\left(a_{1} / c, a_{2} / c\right)$ (or, resp., $\left(c / a_{1}, c / a_{2}\right)$ ) where $a_{i} \in\{a, b\}$. In this last case $\varphi$ must be $c \leqslant a_{i}$ (resp. $a_{i} \leqslant c$ ) which is easily achieved by $\left(a_{2} / c \mid a=b\right)$ (resp. $\left.\left(a_{2} / c \mid a=b\right)\right)$.

We extend the definition of $={ }_{a, b}$ to predicates: $\varphi={ }_{a, b} \psi$ iff $\varphi$ and $\psi$ differ only by $a, b$ swaps. Lemma 57 can be slightly generalised:

Lemma 58. If $P={ }_{a, b} Q, \varphi={ }_{a, b} \psi$ then $\varphi_{P \mid a=b}=\psi_{Q \mid a=b}$.

Proof: By Lemma 57 we only have to prove that if $R=$ $S \mid a=b$ then $R \triangleright \varphi$ implies $R \triangleright \psi$, which is easy, since for each case there is a rule of Definition 30 that uses either $a / b$ or $b / a$ to replace one $a$ with a $b$ or vice versa.

Lemma 59. If $P={ }_{a, b} Q$ then $(P \mid a=b) \sim_{\mathrm{bn}}(Q \mid a=b)$.

Proof: Let $\mathcal{R}$ be the corresponding relation, quantifying over every $P$ and $Q$. We prove that $\mathcal{R}$ is a $\sim_{\text {bn }}$-bisimulation:

1) invariance under arcs is trivial;

2) is implied by Lemma 57;

3) we use Lemma 58 to ensure the communication is possible (when $\mu=\tau$ ) or that the subject of $\mu$ can be related to the subject of the prefix (when $\mu \neq \tau$ ). The resulting processes are still related through $={ }_{a, b}$ since this relation commutes with $\equiv$ and contexts.

We conclude by symmetry of $={ }_{a, b}$.

Lemma 60. If $P$ and $Q$ are prefix-free, and if their preorders coincide on free names, then $P \sim_{\text {bn }} Q$.

Proof: The corresponding relation is a $\sim_{\mathrm{bn}}$-bisimulation: all condition checks are straightforward, even when we add arcs since Definition 30 is compositional: $\operatorname{preor}(P \mid Q)$ only depends on preor $(P)$ and $\operatorname{preor}(Q)$.

Lemma 61. For every fusion process $P$ if $\llbracket P \rrbracket \triangleright a \leqslant b$ or $\llbracket P \rrbracket \triangleright a \curlyvee b$ then $\llbracket P \rrbracket \triangleright a=b$ and $P \equiv P \mid a=b$ (i.e. $a$ and $b$ are related through $P$ 's fusions).

Proof: First we prove that $\llbracket P \rrbracket \triangleright a \leqslant b$ implies $\llbracket P \rrbracket \triangleright b \leqslant a$ by induction on the derivation of the first judgement. The only interesting case is when we use an $\operatorname{arc} b / a$ : then we know that there is the other arc $a / b$ next to $b / a$, so this is enough. We also know that this is coming from $a=b$ in the original process. Now if the hypothesis is about $a \curlyvee b$ we know that there is a name $u$ such that $a \leqslant u$ and $b \leqslant u$ and we use the first part of the proof to prove $u \leqslant a$ and $u \leqslant b$ which you can compose to get $a \leqslant b$ and $b \leqslant a$.

Statement of Theorem 22: Suppose $P$ and $Q$ are processes of the fusion calculus.

1) If $P \equiv Q$ then $\llbracket P \rrbracket \simeq$ bn $\llbracket Q \rrbracket$;

2) if $P \longrightarrow_{\mathrm{EF}} P^{\prime}$ then $\llbracket P \rrbracket \longrightarrow{ }_{\mathrm{bn}} \approx_{\mathrm{bn}} \llbracket P^{\prime} \rrbracket$;

3) conversely, if $\llbracket P \rrbracket \longrightarrow_{\mathrm{bn}} Q$, then $Q \approx_{\mathrm{bn}} \llbracket P^{\prime} \rrbracket$ for some $P^{\prime}$ such that $P \longrightarrow_{\mathrm{EF}} P^{\prime}$.

Proof: 1) Thanks to Theorem 21, it is enough to prove $\llbracket P \rrbracket \sim_{\text {bn }} \llbracket Q \rrbracket$, which we do by induction on the derivation of $P \equiv Q$. The standard base cases like associativity are translated directly into structural congruent processes that are therefore related through $\sim_{\mathrm{bn}}$. The other base cases that those dedicated to fusions:

- $\llbracket a=b\left|P \rrbracket \sim_{\mathrm{bn}} \llbracket a=b\right| P\{a / b\} \rrbracket$ by Lemma 59,

- $\llbracket a=b\left|b=c \rrbracket \sim_{\mathrm{bn}} \llbracket a=c\right| b=c \rrbracket$ by Lemma 59,

- $\llbracket a=b \rrbracket \equiv \llbracket b=a \rrbracket$ by commutativity of $\mid$,

- $\llbracket a=a \rrbracket \sim_{\text {bn }} \llbracket 0 \rrbracket$ by Lemma 60 ,

- $\llbracket(\boldsymbol{\nu} a) a=b \rrbracket \sim_{\text {bn }} \llbracket 0 \rrbracket$ by Lemma 60 .

We conclude thanks to the fact that $\sim_{b n}$ is a congruence and an equivalence relation.

2) Thanks to 1) and the fact $\longrightarrow_{\text {bn }}$ is stable by active contexts we only consider the base case of the reduction relation: $R \stackrel{\text { def }}{=} \bar{a} b . P|a c . Q \longrightarrow \mathrm{EF} b=c| P \mid Q \stackrel{\text { def }}{=} R^{\prime}$. Since $\approx_{\mathrm{bn}}$ is stable by $\equiv$ and active contexts, we just have to consider the following: $\llbracket R \rrbracket \longrightarrow_{\text {bn }}(\boldsymbol{\nu} w y)(b / c|w / y| w b . \llbracket P \rrbracket \mid$ $\bar{y}\langle c\rangle . \llbracket Q \rrbracket)$ which has only one deterministic reduction to $\llbracket R^{\prime} \rrbracket \mid(\boldsymbol{\nu} w y)(w / y)$ which is strongly bisimilar to $\llbracket R^{\prime} \rrbracket$ by Lemma 60.

$3)$ In $\llbracket R \rrbracket$ the only visible prefixes $\pi$. $P$ are the form $\llbracket \pi^{\prime} . P^{\prime} \rrbracket$. Suppose that $\llbracket R \rrbracket \longrightarrow_{\text {bn }} S$ comes from the communication between $\pi_{1} . P$ and $\pi_{2} . Q$ of subjects $a$ and $b$. We know that $\llbracket R \rrbracket \triangleright a \curlyvee b$ which means thanks to Lemma 61 that the communication is possible between $\pi_{1}^{\prime} \cdot P^{\prime}$ and $\pi_{2}^{\prime} \cdot Q^{\prime}$ : for some $R^{\prime}, R \longrightarrow{ }_{\mathrm{EF}} R^{\prime}$. The process $S$ is then one step away to create the next step and free arcs (corresponding to the encoding of the fusion just created) the continuations $\llbracket P^{\prime} \rrbracket$ and $\llbracket Q^{\prime} \rrbracket$ which places us into a situation similar to 2 ).

\section{REFERENCES}

[1] B. Pierce and D. Sangiorgi, "Typing and subtyping for mobile processes," Math. Str. in Comp. Sci., vol. 6, no. 5, pp. 409-453, 1996.

[2] N. Kobayashi, "Type systems for concurrent programs," in 10th Anniversary Colloquium of UNU/IIST, ser. LNCS, vol. 2757. Springer, 2003 , pp. 439-453.

[3] _ "A new type system for deadlock-free processes," in CONCUR, ser. LNCS, vol. 4137. Springer, 2006, pp. 233-247.

[4] K. Honda, V. T. Vasconcelos, , and M. Kubo, "Language primitives and type discipline for structured communication-based programming," in ESOP, ser. LNCS, vol. 1381. Springer, 1998, pp. 122--138.

[5] D. Sangiorgi and D. Walker, The Pi-Calculus: a theory of mobile processes. Cambridge University Press, 2001.

[6] J. Parrow and B. Victor, "The fusion calculus: expressiveness and symmetry in mobile processes," in LICS. IEEE, 1998, pp. 176 -185.

[7] — "The update calculus (extended abstract)," in AMAST, ser. LNCS, vol. 1349. Springer, 1997, pp. 409-423.

[8] L. Wischik and P. Gardner, "Explicit fusions," Theor. Comput. Sci., vol. 340, no. 3, pp. 606-630, 2005.

[9] Y. Fu, "The $\chi$-calculus," in APDC. IEEE Comp. Soc., 1997, pp. 74-81.

[10] C. Laneve and B. Victor, "Solos in concert," Math. Str. in Comp. Sci., vol. 13 , no. 5, pp. 657-683, 2003.

[11] P. Gardner and L. Wischik, "Explicit fusions," in MFCS, ser. LNCS, vol. 1893. Springer, 2000, pp. 373-382.

[12] J. Parrow and B. Victor, "The tau-laws of fusion," in CONCUR, ser. LNCS, vol. 1466. Springer, 1998, pp. 99-114.

[13] G. L. Ferrari, U. Montanari, E. Tuosto, B. Victor, and K. Yemane, "Modelling Fusion Calculus using HD-Automata," in CALCO, ser. LNCS, vol. 3629. Springer, 2005, pp. 142-156.

[14] F. Bonchi, M. G. Buscemi, V. Ciancia, and F. Gadducci, "A presheaf environment for the explicit fusion calculus," J. Autom. Reasoning, vol. 49, no. 2, pp. 161-183, 2012.

[15] M. Boreale, M. G. Buscemi, and U. Montanari, "A general name binding mechanism," in TGC, ser. LNCS, vol. 3705. Springer, 2005, pp. 61-74.

[16] N. Kobayashi, B. Pierce, and D. Turner, "Linearity and the pi-calculus," TOPLAS, vol. 21, no. 5, pp. 914-947, 1999.

[17] K. Honda and N. Yoshida, "On reduction-based process semantics," Theor. Comp. Sci., vol. 152, no. 2, pp. 437-486, 1995. 
[18] R. De Nicola and M. Hennessy, "Testing equivalences for processes," Theor. Comput. Sci., vol. 34, pp. 83-133, 1984.

[19] D. Sangiorgi, "Pi-calculus, internal mobility, and agent-passing calculi," Theor. Comput. Sci., vol. 167, no. 1\&2, pp. 235-274, 1996

[20] M. Merro, "Locality in the pi-calculus and applications to distributed objects," Ph.D. dissertation, École des Mines, France, 2000.

[21] J. Bengtson, M. Johansson, J. Parrow, and B. Victor, "Psi-calculi: Mobile processes, nominal data, and logic," in LICS. IEEE, 2009, pp. 39-48.

[22] B. Victor, "The fusion calculus : Expressiveness and symmetry in mobile processes," Ph.D. thesis, Uppsala University, 1998.

[23] Web appendix to this paper, available at http://hal.inria.fr/hal-00818068, 2013.

[24] H. Hüttel, "Typed $\psi$-calculi," in CONCUR, ser. LNCS, vol. 6901. Springer, 2011, pp. 265-279. 